\title{
Evaluation of Consultations Requested from Patients Admitted to the Emergency Department
}

\author{
Enver Özçete', Ömer Kaçmaz², Ömer Damar², Mehmet Üstündağ², Murat Orak², Cahfer Güloğlu² \\ 'Department of Emergency Medicine, Ege University Faculty of Medicine, İzmir, Turkey \\ 2Department of Emergency Medicine, Dicle University Faculty of Medicine, Diyarbakır, Turkey
}

\begin{abstract}
Aim: This study was aimed to conduct an investigation towards evaluation of the efficiency of the consultation system that is in place in the emergency service of our hospital and identification and correction of deficiencies in this system.

Materials and Methods: Patients who were admitted to the emergency department and for whom requesting a consultation were required for any reason were included in the study. Forms were filled out by the emergency research assistants. Patients whose data was incomplete and consents could not be obtained were excluded from the study.

Results: A total of 213 patients were included in the study, and a total of 315 consultations were requested. The total number of consultations requested by the internal medicine departments was 126 , and the total number of consultations requested by the trauma (surgery) departments was 189 . Of the 213 patients, 104 (48.8\%) were hospitalized and 109 (51.2\%) were discharged. The average number of consultations of 213 patients was $1.5 \pm 0.7$, the average length of stay of the patients in the emergency service was $366.7 \pm 312.9 \mathrm{~min}$. and the mean age of the patients was $41 \pm 24$ years. The average seniority of the consultant physicians in a total of 315 consultations performed was $26.8 \pm 10.6$ months, and the average consultation response time of the consultant physicians was $62.2 \pm 67.8$ min.
\end{abstract}

Conclusion: Because of the changing healthcare system in our country, the consultation services, particularly in university hospitals, should be re-structured. (Eurasian J Emerg Med 2015; 14: 113-7)

Keywords: Emergency department, consultation, consultation time

\section{Introduction}

Consultation is a shared and essential factor in the departmental function of emergency medicine (1,2). An emergency consultation is most common for patient admission. The emergency consultations are evaluation by same visit advice or interventions by the consultant who has a specific level of expertise (3).

Although it cannot be determined with clear boundaries in which cases and from which specialities a consultation will be requested, it is recommended that every hospital establishes a consultation protocol and control system in compliance with its structure to minimize the problems encountered in practice $(4,5)$. The emergency medicine and hospital management and other relevant departments should together identify the rules of such a protocol. Notwithstanding that it is one of the physicians' rights, the right of a physician for consultation provides an advantage for patient health if only used aptly, timely, and appropriately (6).
This study was aimed to conduct an investigation towards evaluation of the efficiency of the consultation system that is in place in the emergency department of our hospital and identification and correction of deficiencies in this system.

\section{Materials and Methods}

After the approval of the University Hospital Ethics Committee is obtained, patients who were admitted to the Department of Emergency Medicine of the University School of Medicine and for whom requesting a consultation was required for any reason were included in this prospective and descriptive study. The study was conducted between February and June 2013. Parameters such as patient's diagnosis, age, gender, complaints for admission, mode of admission, laboratory tests and imaging examinations requested, department consulted, consultation response times, whether or not the consulting physician consulted the academic emergency medicine physician in charge,

Correspondence to: Enver Özçete e-mail: eozcete@gmail.com 
form of requesting consultation, patients' length of stay in the emergency department, and admission/discharge of patients were written in the forms prepared for the patients included in the study. The research assistants were informed on how to complete the forms for participating in the case prior to the study. Forms were filled out by the emergency research assistants. Patients whose data was incomplete and consents could not be obtained were excluded from the study.

After the forms were collected, the groups were created based on the length of stay in the emergency department of patients with internal or trauma complaints according to their initial complaints, patients with one or more than one consultation required, patients discharged or admitted, and patients for whom the academic emergency medicine physician in charge was consulted or not (via telephone consultation).

The length of stay in the emergency department of the patients was determined based on the difference between the admission to and discharge from the hospital, and the patients were divided into three groups. The average consultation time is the difference between the time the consulting physician was informed about the patient and the consulting physician provided his/her consideration. The group with a small number of consultations requested was created by combining the departments with a total number of consultations (i.e., <10) in the emergency department.

\section{Statistical analysis}

Descriptive statistics (mean), standard deviation, and frequency tables were used for the basic statistical analysis. The continuous variables were expressed as mean \pm standard deviation and the categorical data as median and percentage. The chi-square analysis was used to determine the significance between the double groups in the advanced statistical analysis, and the Student's t-test was used to determine the significance between the averages.

\section{Results}

A total of 213 patients were included in the study and a total of 315 consultations were requested. The total number of consultations of the internal medicine departments was 126, and the total number of consultations of the trauma (surgery) departments was 189 . While 85 of the patients included in the study were female with a mean age of $38.8 \pm 23.6$ years, 128 of them were male with a mean age of $42.4 \pm 24.2$ years. There was no statistically significant difference between the female and male patients.

Of the 213 patients, 104 (48.8\%) were hospitalized and 109 (51.2\%) were discharged. The average number of consultations of 213 patients was $1.5 \pm 0.7$, the average length of stay in the emergency department of the patients was $366.7 \pm 312.9 \mathrm{~min}$. and the mean age of the patients was $41 \pm 24$ years. The average seniority of the consultant physicians in a total of 315 consultations performed was $26.8 \pm 10.6$ months, and the average consultation response time of the consultant physicians was $62.2 \pm 67.8 \mathrm{~min}$. The consultants consulted to the academic emergency medicine physician in charge for $144(45.7 \%)$ of 315 consultations requested, while 171 of these consultations were not referred and evaluated by the consultants. Of the 213 patients, 117 were evaluated by a single consultant and 96 of them were evaluated by more than one consultant.

According to Table 1, the departments with the highest number of consultations requested were the internal medicine and orthopae- dics departments with 48 consultations. The seniority of the physicians from the internal medicine and general surgery departments called for a consultation was greater than the seniority of the physicians from the other departments.

The average consultation response time of the physicians from the department of neurology was shorter than the other departments and the mean age of patients was higher.

The average number of patients who received a consultation from the neurosurgery department was higher than the other departments, and the average seniority of the physicians called for a consultation was lower than the physicians from the other departments.

The mean age of patients who received an orthopaedic consultation was lower than the patients from the other departments.

The length of stay in the emergency department of the patients from the plastic surgery department was shorter than the other departments, and the average age of these patients was higher, while their average consultation rates were lower.

The seniority of the physicians from the department of otolaryngology was lower than the physicians from the other departments.

Ninety of 213 patients applied because of trauma complaints, while 123 of them applied because of internal complaints.

When the patients with trauma complaints were compared to the patients with internal complaints, patients of the neurosurgery and orthopaedic departments consisted of the patients with trauma complaints and patients of the general surgery, neurology, and plastic surgery departments consisted of the patients with internal complaints.

It was found that the rate of patients with trauma complaints was greater among male patients, and the rate to bring these patients to the emergency department by an ambulance was higher.

It was revealed that patients with internal complaints admitted to the emergency department as outpatients and biochemical parameters such as complete blood count, biochemistry, blood gas, and cardiac enzymes were requested more for such patients.

The number of direct radiography and computed tomography scans was higher in patients with trauma complaints.

When the groups created based on the patients' length of stay in the emergency department were compared, it was found that the number of patients with internal complaints was higher in patients from the group created based on the length of stay of 240-480 min.

The number of consultations received from the department of surgery for patients with trauma complaints was greater.

There was no statistically significant difference between other parameters.

It was found that the academic emergency medicine physician in charge was consulted more by the internal, neurosurgery, and orthopaedics consultants and less by the plastic surgery and ear, nose, and throat (ENT) consultants in the groups created with respect to consultation to the academic emergency medicine physician in charge.

The academic emergency medicine physician in charge was consulted more for patients brought to the emergency department by an ambulance and less for patients admitted to the emergency department as outpatients.

The rate for consulting the academic emergency medicine physician in charge was lower in consultations requested from the surgical department. 
Table 1. Distribution of consultations by the departments

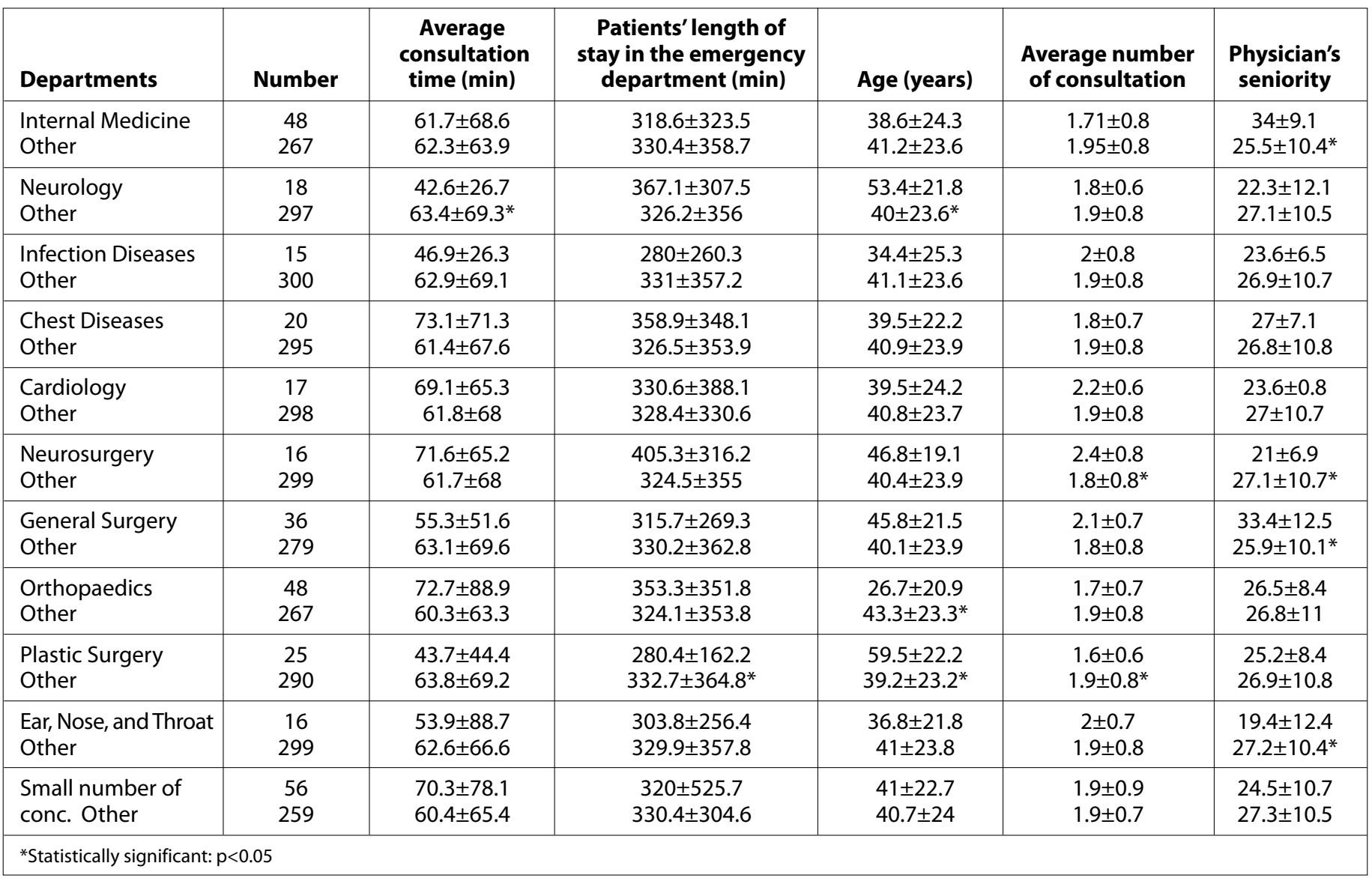

When the group in which patients requested one consultation was compared to the group in which patients requested more than one consultation, the rate for requesting one consultation was higher in patients receiving a consultation from the internal medicine department. The rate for requesting more than one consultation was higher in patients receiving a consultation from the neurosurgery and general surgery departments.

The rate for requesting one consultation was higher in outpatients. The rate for requesting a complete blood count, biochemistry, blood gas, direct radiography, ultrasonography, and computed tomography was higher in the group with more than one consultation requested. The rate for requesting a consultation via a mobile phone was higher in the group with more than one consultation requested.

When the patients discharged from and hospitalized in the emergency department were compared, the number of patients hospitalized by the internist and orthopaedic consultants was higher than the number of patients hospitalized by the other consultants.

The rate of discharge by the ENT consultants was higher than the other consultants. Patients receiving blood products were hospitalized for a longer period of time.

When Table 2 was examined, it was understood that the average seniority of the consultant physicians of patients hospitalized was higher than the average seniority of the consultant physicians of patients discharged.

The average length of stay in the emergency department of the patients with one consultation requested was shorter than the average length of stay in the emergency department of the patients with more than one consultation requested.
The average consultation response time of the consultant physicians was longer in patients from the surgery group than in patients from the internal medicine group. The average age of patients from the surgery group was lower than patients from the internal medicine group.

In patients from the group created based on the patients length of stay in the emergency department for a period of 0-240 $\min$,

* The rate of admission to the surgical department and requesting a direct radiography was higher.

* The rate for requesting a complete blood count and biochemical and blood gas analysis was lower.

* The rate for requesting a computed tomography and ultrasonography was lower.

* The rate of evaluation of patients by one consultant was higher. In patients from the group who stayed in the emergency department for a period of 240-480 min,

* The rate of admission to the internal medicine department was higher and to the surgery department was lower.

* The rate for requesting an analysis of the complete blood count, biochemistry, blood gas, cardiac enzymes, and ultrasonography was higher.

* The rate of patients with internal disease complaints was higher.

* The rate for requesting a computed tomography for patients staying in the emergency department for a period above 480 min was higher, and the other parameters were not significantly different. 
Table 2. Mean values by variables

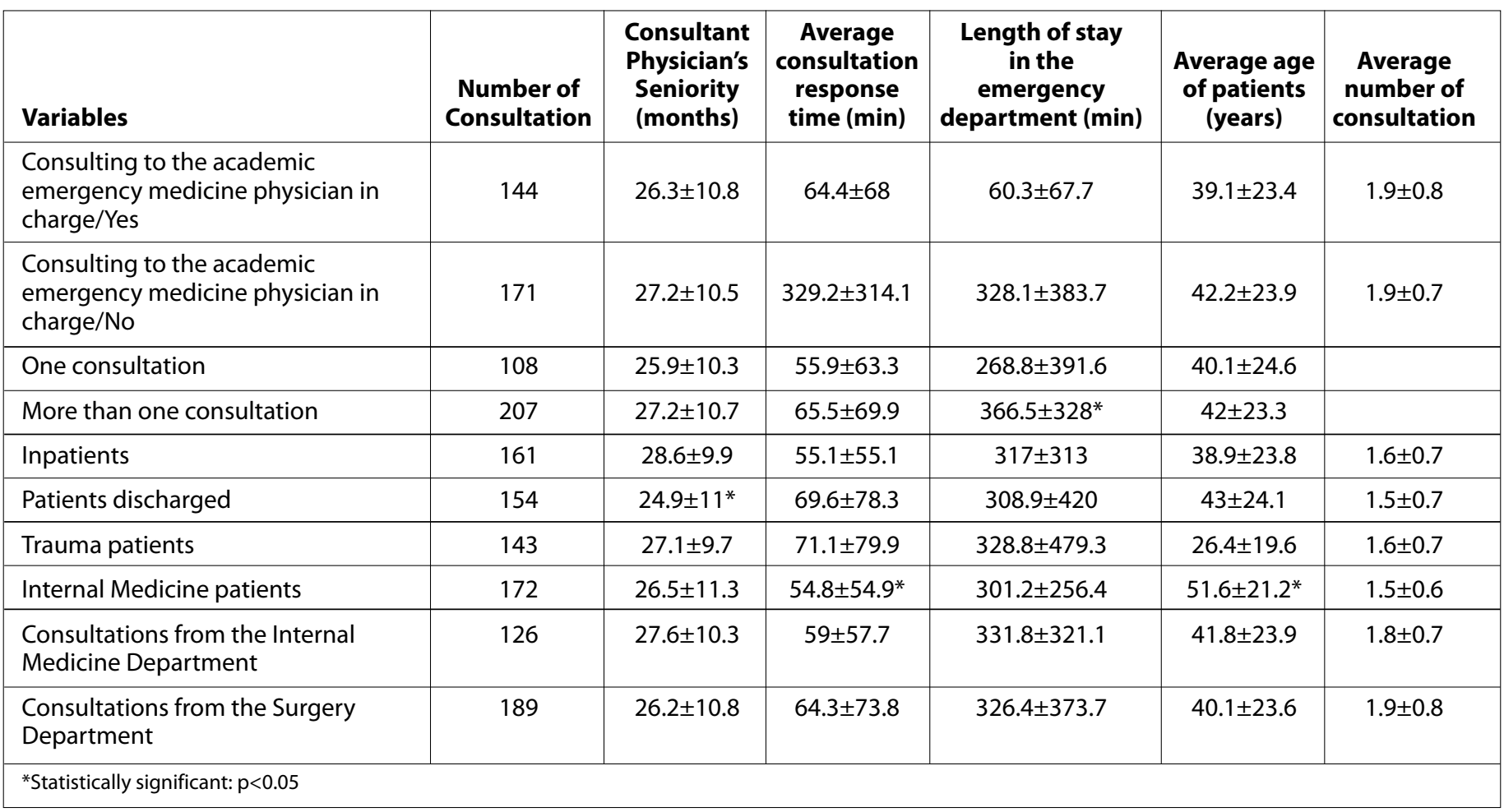

\section{Discussion}

When the literature was reviewed, it was determined in the study performed by Karakaya et al. (7) that the internal medicine (72; $21.1 \%)$, the gynaecology and obstetrics $(47 ; 13.7 \%)$, and the general surgery departments $(35 ; 10.2 \%)$ were the most frequently consulted departments. The least consulted departments were found to be psychiatry $(4 ; 1.2 \%)$, anaesthesiology $(6 ; 1.8 \%)$, and ophthalmology $(6 ; 1.8 \%)(8)$. Another study found the most frequently consulted departments to be cardiology, orthopaedics and traumatology, internal medicine, and general surgery (9). The most frequently consulted departments in our study were consistent with the other studies, despite the fact that these studies were old (2002-2003).

The patients' average length of stay in the emergency department varies in studies performed; the average length of stay in the emergency department of the patients with consultation requested was $2 \mathrm{~h} 31 \mathrm{~min}$ in the study performed by Karakaya et al. (7), the patients' length of stay in the hospital was $2 \mathrm{~h}$ in the study performed by Kılıçaslan et al. (8), and the patients' average length of stay in the emergency department was $3.3 \mathrm{~h}$ in the study performed by Oktay et al. (9) In a study conducted in California (10), the average length of stay was found to be $56 \mathrm{~min}$. The average length of stay was 3.2 $\mathrm{h}$ in the United States in 2002 according to the data from the Centre for Disease Control and Prevention, and approximately two-thirds of the emergency patients waited for 1-6 h in the emergency department (11). The patients' length of stay in the emergency department is highly variable according to the literature. The length of stay in the studies conducted in Turkey was much shorter than our study. Data from the studies conducted in Turkey dates back to 2002-2003. We are of the opinion that the length of patient follow-up by the emergency physicians and consultant physicians was prolonged for different reasons such as increased number of patients because of the commencement of provision of the healthcare services in our country's healthcare system for patients without a mandatory professional insurance and particularly for patients from all institutions in the University hospitals without a referral chain from 2003, and the patients' length of stay in the emergency department has extended because of the lack of empty beds in hospitals. There was no statistically significant difference between the patients discharged and patients hospitalized in our study with respect to the length of stay in the hospital.

It was understood from the study by Karakaya et al. (7) that 221 (80.1\%) of 276 patients were hospitalized in the intensive care unit or other services. The proportion of hospitalizations for those patients receiving specialist consultations is highly variable. Cortazzo et al. (12) reported that $87 \%$ of all consulted patients were admitted, and Woods et al. (13) reported that a proportion of $54 \%$ were admitted. Curry and Wang (14) reported an admission rate of $68 \%$ for the highest acuity site and $64 \%$ for the lowest acuity site. The rate of hospitalization in our study was lower than the other studies. The reason for this may be the increased number of patients admitted to the emergency department of the University hospital compared to past studies and prolonged length of follow-up in the emergency department and discharge from the emergency department because of the lack of empty beds in hospitals.

Guidelines from professional organisations suggest that a reasonable response time of consultants to the ED is between 30 and $45 \mathrm{~min}$, although it is primarily dependent on the current needs of the patient (15).

The average duration of consultations in our study was longer than the other studies. It was planned to shorten the duration of consultations in our hospital by taking the necessary measures.

It was determined in the study performed by Oktay et al. (9) that 4441 patients (74.9\%) requested one consultation and 1488 patients 
(25.1\%) requested more than one consultation. The rate of evaluation by one consultant physician in our study was considerably reduced. Although this situation can vary according to the hospitals' facilities, we can say that the rate for requesting one and more than one consultation has increased today, considering the increased rate of consultations. The increase in the rate for requesting one and more than one consultation extends the patients' length of stay in the emergency department and this situation leads to unintended consequences for both patients and physicians.

The fact that the academic emergency medicine physician in charge was consulted did not create a statistically significant difference in the patients' parameters evaluated. We believe that the physicians who evaluate emergency department patients at their bedside are very important. The patients' rate of hospitalization increased as the consultant physician's seniority increased in our study. We are of the opinion that consultations in the emergency department should be performed by a specialist or by the most experienced physician in the department from which a consultation is requested.

When the patients' length of hospitalization were investigated, it was found that performance of an evaluation by a consultant in the department that the patients were admitted to, types of blood tests conducted, and imaging requested from the patients was effective. The rate for requesting a tomography was higher in the patients who stayed the longest in the emergency department, and patients from this group were the patients who were brought to the emergency department with trauma complaints by an ambulance and who were admitted to the resuscitation department and were evaluated by more than one consultant. We believe that the length of follow-up of these patients was prolonged because these patients are often multiple trauma cases. Patients who stayed the shortest in the emergency department were the patients from the group that were admitted to the surgery department and were evaluated with a direct radiography and by one consultant and for which other tests were requested less. We believe that the diagnosis and treatment of these patients have been concluded in a short time because these patients generally involve a single department. The rate of admission to the internal medicine department and requesting blood tests and ultrasonography for the patients from the group who stayed in the emergency department for 240-480 min was higher. We believe that the length of stay of these patients in the emergency department has prolonged because of waiting for the results of blood tests and ultrasonography requested for these patients and performance of control examinations, where necessary.

\section{Study limitations}

Our study was conducted at a University hospital. Therefore, information about the consultations in other hospitals could not be provided. Further consultation multicenter studies are required.

\section{Conclusion}

Because of the changing healthcare system in our country, the number of patients in the emergency departments of University hospitals in particular, consultation rates, rate of hospitalization, and patients' length of stay in the emergency department have changed. Therefore, the consultation departments should be re-structured.

Ethics Committee Approval: Ethics committee approval was received for this study from the ethics committee of Dicle University Faculty of Medicine.

Informed Consent: Written informed consent was obtained from patients who participated in this study.

Peer-review: Externally peer-reviewed.

Conflict of Interest: No conflict of interest was declared by the authors.

Financial Disclosure: The authors declared that this study has received no financial support.

\section{References}

1. Rosen P. Emergency department disposition and knowledge of other specialties. J Emerg Med 1986; 4: 325-6. [CrossRef]

2. Tintinalli JE, McCall K. Importance of emergency physicians as referral sources for academic medical centers. Ann Emerg Med 1994; 23: 65-9. [CrossRef]

3. Guertler AT, Cortazzo JM, Rice MM. Referral and consultation in emergency medicine practice. Acad Emerg Med 1994; 1: 565-71. [CrossRef]

4. La Coursiere DY, Nager CW. A comparison of two models of gynecology service consultation to the emergency department in an academic medical center. Am J Obstet Gynecol 2003; 188: 1166-8. [CrossRef]

5. Vissers MC, Hasman A, vd Linden CJ. Consultation behaviour of residents supported with a protocol processing system (ProtoVIEW) at the emergency ward. Int J Biomed Comput 1995; 38: 181-7. [CrossRef]

6. The Turkish Medical Association (TMA), Code of Medical Ethics January 2002: 24.

7. Karakaya Z, Gökel Y, Açıkalın A, Karakaya O. Evaluation of the process and effectiveness of consultation system in the Department of Emergency Medicine. Ulus Travma Acil Cerrahi Derg 2009; 15: 210-6.

8. Kılıçaslan I, Bozan H, Oktay C, Göksu E. Demographic characteristics of patients admitted to the emergency department in Turkey. Eurasian J Emerg Med 2005; 5: 5-13.

9. Oktay C, Cete Y, Eray O, Pekdemir M, Gunerli A. Appropriateness of emergency department visits in a Turkish university hospital. Croat Med J 2003; 44: 585-91.

10. Lambe S, Washington DL, Fink A, Laouri M, Liu H, Scura Fosse J, et al. Waiting times in California's emergency departments. Ann Emerg Med 2003; 41: 35-44. [CrossRef]

11. McCaig LF, Burt CW. National Hospital Ambulatory Medical Care Survey: 2002 emergency department summary. Adv Data 2004: 1-34.

12. Cortazzo JM, Guertler AT, Rice MM. Consultation and referral patterns from a teaching hospital emergency department. Am J Emerg Med 1993; 11: 456-9. [CrossRef]

13. Woods RA, Lee R, Ospina MB, Blitz S, Lari H, Bullard MJ, et al. Consultations in the emergency department: exploring rates and complexity. Can J Emerg Med 2006; 8: 204.

14. Curry DG, Wang DM. Variation of consultation practice in urban emergency physicians. Can J Emerg Med 2006; 8: 182-3.

15. Reid C, Moorthy C, Forshaw K. Referral patterns: An audit into referral practice among doctors in emergency medicine. J Emerg Med 2005; 22: 355-8. [CrossRef] 